\title{
La relevancia del grupo en la dinámica institucional: Un equipo de buceo universitario
} The relevance of the group in the institutional
dynamics: A university diving team

\author{
Marcela Victoria Rendón Cobián* \\ Universidad Autónoma Metropolitana Iztapalapa, \\ Ciudad de México, México \\ ORCID: https://orcid.org/0000-0002-1828-9039
}

DoI: http://dx.doi.org/10.28928/revistaiztapalapa/812016/atc2/rendoncobianmv

\section{Resumen}

El objetivo de este trabajo es destacar la importancia del grupo en la dinámica institucional. El grupo constituye un dispositivo generador de sentido social a la vez que se conforma como un mecanismo que proporciona cobijo psicológico a sus integrantes y funciona también como instrumento racional para la ejecución de la tarea. Se ilustra el debate teórico con el estudio del Equipo de Buceo de Ciencias de la Facultad de Ciencias de la Universidad Nacional Autónoma de México. Se trata de una investigación cualitativa, basada en entrevistas semiestructuradas y observación participante. Una de las principales conclusiones del trabajo reside en la consideración de que el grupo es una unidad caracterizada por el entrecruzamiento de lógicas de acción diversas, y de ese modo conforman un complejo entramado social de convergencias y contradicciones que propician tanto la acción social como un conjunto potencial de conflictos. El grupo es un espacio social siempre en tensión.

Palabras claveः grupo, institución, organización, buceo, universidad.

* Universidad Autónoma
Metropolitana Iztapalapa
marcela_uami@hotmail.com

\section{IZTAPALAPA}

Agua sobre lajas 


\begin{abstract}
The aim of this paper is to highlight the importance of the group in the institutional dynamics. The group is a social generator device, a mechanism that provides psychological shelter to its members and a rational tool for the execution of the task. Theoretical discussion is illustrated by the study of the Sciences Diving Team of the Faculty of Sciences at the National Autonomous University of Mexico. This is qualitative research based on semi-structured interviews and participant observation. One of the main conclusions of this paper is that the group is considered a unit characterized by the intermingling logic of various actions. These make up a complex social fabric of convergences and contradictions that encourages both social action and potential conflicts. The group is a social space that always experiences tension.
\end{abstract}

Key words: group, institution, organization, diving, University. 
E objetivo central de este trabajo es destacar la importancia del grupo en la dinámica institucional, así como analizar su desempeño tomando en consideración que este constituye un espacio socioafectivo encargado de realizar un conjunto de tareas en un ambiente organizacional. Se aborda el estudio de la relación, siempre compleja, de la institución con la organización y se analizan las características principales de los distintos tipos de grupo, así como los fines y motivos que poseen los individuos para integrarse en una acción colectiva acotada. Estos buscan generar sentido a su existencia social mediante la suscripción a diversos ámbitos institucionales, los cuales se concretan en espacios organizacionales y grupales. Así, el individuo resulta beneficiado cuando un grupo logra una cierta inserción social, mejor desempeño en sus actividades y apoyo intersubjetivo, aunque ello propicie restricciones a su libertad a causa de las normas grupales y las reglas formales impuestas por la organización, provocando con ello un cierto desencanto ante los resultados obtenidos. El trabajo en grupo genera sentido social, proporciona abrigo psicológico a sus integrantes y permite logros que no se tendrían de manera individual, además de potenciar el nivel de desempeño.

En el trabajo de campo se aborda el estudio de un grupo de buceo universitario. Se destacan los motivos que tienen los individuos para involucrarse ampliamente en la realización de sus tareas. El grupo constituye un mecanismo para el logro de objetivos instrumentales mediante la división del trabajo, pero también conforma un universo simbólico y afectivo en sí mismo. Este estudio de caso nos permite hacer más visibles y entrelazar estos tres ámbitos del grupo -social, instrumental y afectivo-, al tratarse de un colectivo de jóvenes universitarios que realizan tareas especializadas de manera totalmente voluntaria y con un alto contenido de riesgo.

Este trabajo se compone de dos apartados, el primero se refiere a los aspectos teóricos. Se abordan en él los conceptos de institución, organización y grupo, y se analizan sus diferentes tipos. Se estudian conceptos centrales del funcionamiento grupal como su relación con la institución y la organización, así como su contribución a la elaboración de la identidad mediante mecanismos simultáneos de integración y diferenciación. El segundo apartado inicia con una breve explicación de 
las herramientas metodológicas utilizadas en la investigación para abordar posteriormente el caso de un grupo universitario de buceo, constituido por jóvenes que desempeñan y viven de manera intensa tanto la actividad sustantiva del grupo como sus relaciones afectivas. Se constata que el grupo es parte, en ocasiones en forma poco clara y diversa, de una organización que mantiene internamente relaciones con distintas instancias. Un tema central que se analiza es la relación, en ocasiones complementaria y en otras contradictoria, que se establece entre lo que se considera el grupo y el equipo de trabajo. En la parte final, y a manera de conclusión, se expresan algunas ideas que pretenden contribuir a una mejor comprensión de los grupos, tratando de resaltar la necesidad tanto de ubicar dicha discusión en un lugar más central de la reflexión social y organizacional como de escapar a las estrecheces que encasillan el estudio de caso a una especie de anécdota local irrepetible.

\section{Elementos teóricos}

\section{De la institución, la organización y el grupo}

El estudio del grupo ha adquirido una importancia creciente desde el siglo pasado al ser abordado por un número importante de disciplinas, tales como la psicología social, la sociología, el psicoanálisis, la antropología social y, posteriormente, los estudios organizacionales. De acuerdo con Maisonneuve (1980), un autor reconocido por sus aportaciones a la dinámica de grupos, existen dos tipos de factores que se observan en la construcción de la cohesión grupal, los que provienen del contexto externo y los propios del interno. Los primeros son aquellos que ya existían antes de que se formara el grupo, como pueden ser las normas y reglas que rigen a las diversas instituciones de la sociedad como la familia, la empresa, el hospital, o también cuando un grupo forma parte de otro de mayor tamaño. En este mismo sentido, señala Lapassade que el encuentro del grupo con el ámbito institucional es siempre ineludible:

La experiencia inmediata de la vida social se sitúa siempre en los grupos: la familia, la clase, los amigos. En el trabajo son siempre los grupos los que se encuentran en el horizonte inmediato de la experiencia: es el equipo en la empresa, es el grupo sindical. Pero ya, en esas organizaciones, un nuevo elemento aparece rápidamente: el grupo se encuentra inmerso en un sistema institucional (Lapassade, 2006: I). 
El concepto de institución ha sido uno de los más utilizados en las ciencias sociales y las humanidades; sin pretender entrar en detalle en el amplio debate actual que ha suscitado, podemos señalar que la institución es un recorte imaginario del orden social que implica un alto nivel de idealidad (Montaño, 2015) y establece expectativas de comportamiento mediante el concepto de rol $^{1}$ (Berger y Luckman, 1968). Al respecto, Kaës plantea la siguiente definición:

La institución. La defino como el orden por el cual se funda un colectivo con el objetivo de realizar una tarea útil, necesaria para el funcionamiento social: reproducción de la vida, educación, salud, trabajo, defensa colectiva, sistemas religioso y cultural, basados en representaciones compartidas (Kaës, 2004: 655).

Enriquez (1992) sugiere que el estudio de las organizaciones comporta el análisis de siete instancias, tres de las cuales son relevantes para este trabajo: el grupo, la organización y la institución ${ }^{2}$ La institución implica una perspectiva acerca de la naturaleza humana, la cual se encuentra en el centro de un cierto saber especializado que crea normas de convivencia. Dicha perspectiva, al ser interiorizada por los sujetos, genera en ellos sentidos diversos a su existencia social y hace de la norma social un marco de referencia para su comportamiento; la institución forma parte del aparato psíquico de los individuos. Puede así ser entendida como un dispositivo que permite la movilización de procesos psíquicos en vistas de realizar un conjunto de tareas (Kaës, 2005). La organización, por otro lado, es la concreción, al menos parcialmente, del ideal institucional; la organización es, como dice Enriquez, la institución hecha fierros. La institución prefigura a la organización (Friedland y Alford, 200I, Rendón, 2007) y le proporciona legitimidad social. La instancia grupal, en cuanto a ella, señala Enriquez, constituye una realidad colectiva que si bien está formada por individuos, no puede reducirse a una simple sumatoria; a ellos los reúne una causa común, superior a sus fuerzas individuales. Diversos dispositivos psíquicos son puestos en operación en su interior; uno de los más relevantes es el de la idealidad, el cual reside en el superyó, resultado de la interiorización de la regla

1 Berger y Luckmann acentúan la importancia del rol como forma también concreta, u objetivada, de la noción institucional: "Las instituciones se encarnan en la experiencia individual por medio de los roles, en los que, objetivados lingüísticamente, constituyen un ingrediente esencial del mundo objetivamente accesible para cualquier sociedad" (1968: 98).

2 Las otras instancias propuestas por el autor son la mítica, la socio-histórica, la individual y la pulsional. 
moral, lo que le permite al sujeto discernir entre el bien y el mal, lo correcto y lo indebido, lo bello y lo feo, lo justo y lo injusto, etc. El individuo tiene una imperiosa necesidad de idealizar para comprometerse con los demás. Idealiza distintos ámbitos de su existencia social como son el grupo y la institución. Con respecto al primero, nos recuerda Enriquez:

¿Por qué el individuo acepta idealizar a la sociedad (o al grupo al que pertenece)? Las razones son, de acuerdo con los trabajos de Freud [...] que cada ser humano posee pulsiones, fantasmas contradictorios, que hacen que el individuo busque a través de su vida una identidad estable que lo proteja de la locura, instalada en él, de la angustia de la muerte prometida e ineluctable, que lo confrontan con su propio caos y lo ponen constantemente al borde del abismo (Enriquez, 1997: 345).

Con relación a la idealización de lo social o institucional, el autor afirma que:

Idealizar lo social (o una de sus instituciones) es creer estar a salvo por este objeto maravilloso y lejano que, por el mito colectivo que instaura, lo protege de un trabajo de duelo, le evita la pérdida y el sufrimiento, ubica su psique en un estado sin conflicto; el ideal hace un llamado a identificaciones profundas, procurando como forma de placer el evitar la pérdida $y$, al contrario, el encuentro con la plenitud (Enriquez, 1997: 345).

La relación entre institución y organización no es simple (Rendón, 2007). En lo particular, quisiera destacar la imposibilidad de coincidencia total entre ambas. Si bien la primera es una referencia para la segunda, esta última conoce un margen de maniobra que la aleja de aquella. Esta divergencia, que trastoca el logro de los fines sociales de la organización, es explicada generalmente a partir del fenómeno de la burocratización, entendido como el desplazamiento de los fines y el sobredimensionamiento de los medios, es decir, su conversión en fines acotados a grupos de poder (Michels, 1976). Bleger lo explica muy bien: toda organización tiende a pervertirse, en una especie de ley general: "en todas ellas los objetivos explícitos para los cuales han sido creadas corren siempre el riesgo de pasar a un segundo plano, pasando a un primer plano la perpetuación de la organización como tal" (Bleger, 1970: 7-8).

La burocratización provoca un debilitamiento del vinculo institucional y genera con ello una fuerte sensación de sufrimiento (Kaës, 2005; Cuevas y Guerra, 2010). El grupo puede entonces entrar en contradicción con la organización, cuando se considera que esta no representa la defensa de los ideales institucionales. Algo similar acontece con el burnout, el cual ha sido traducido al español como fatiga emocional 
en el trabajo (Freudenberger, 1974ः 1980). Esta es provocada por la incapacidad que resiente el individuo para alcanzar los fines institucionales debido, entre otros, a la complejidad de la tarea realizada y al proceso mismo de burocratización.

De lo anterior se puede concluir que el grupo se halla inmerso en un conjunto de contradicciones. Por una parte, en el espacio institucional surgen contradicciones internas como aquellas referidas, por ejemplo, a las duplas libertad/obediencia o verdad/felicidad, las cuales generan dilemas éticos, o bien, como lo expresan Bateson y colaboradores (1956), dobles vínculos, es decir, procesos de sujeción contradictoria. Ahora bien, si consideramos que el apego del grupo se realiza de hecho a una multiplicidad simultánea de ideales institucionales -familia, universidad, amigos, iglesia, etc.-, ello provoca también una ambigüedad e incertidumbre en la manera de enfrentar la realidad. Por otro lado, en su relación con la organización, esta le permite al grupo el acceso a cierto tipo de recursos -económicos y políticos, por ejemplo-, contribuye a la realización de su tarea referida institucionalmente, pero también la limita por su propensión burocrática. Finalmente, el grupo encuentra una contradicción sustantiva entre la realización de su tarea y su funcionamiento en tanto dispositivo psíquico inconsciente de resguardo colectivo. Así, el grupo cumple simultáneamente diversas tareas en un espacio amplio de contradicciones; el grupo es a la vez resguardo institucional, dispositivo organizacional y cobijo psicológico.

\section{En torno al grupo}

Resulta pertinente ahora abordar, retomando la propuesta de Maisonneuve (1980), los factores internos en la constitución y dinámica del grupo. De entre ellos quisiera destacar los de corte socioafectivo, que son aquellos que se derivan de la necesidad emocional que tienen los individuos de pertenencia. Los motivos por los que el individuo desea participar en un grupo son diversos; por ejemplo, resulta atractivo integrarse a un grupo cuando este posee cierto poder en alguna esfera social, cuando cuenta con un amplio reconocimiento, o bien cuando permite reforzar el sentido social o ser objeto de cuidado y amor. En todos los casos, el individuo se siente orgulloso de pertenecer a dicho grupo; ello le proporciona una sensación de fortaleza, seguridad, sentido y protección. Existen otros factores internos de naturaleza operativa y funcional que son aquellos que permiten al individuo satisfacer necesidades y alcanzar objetivos de tipo funcional.

El concepto de grupo resulta sencillo cuando se aborda de manera superficial, de esta forma se define como un conjunto de individuos que se reúne para conseguir 
un objetivo común. Una reunión de individuos puede tomar diversas formas. Con el fin de lograr una adecuada distinción entre los diferentes tipos de grupos, retomaré algunas características básicas como la estructuración, la cual consiste en el grado de organización interna, reflejado entre otros en la diferenciación de roles, en el tiempo de duración del grupo, que puede ir desde unas cuantas horas hasta varios años, en el número de miembros que lo componen, en el tipo y la profundidad de relaciones que se establecen entre ellos, en la conciencia que se tiene de los objetivos y los intereses que se persiguen.

Por otro lado, el grupo asume también formas diferentes en función de su ubicación en la sociedad, es decir, de las instituciones que representan el orden social, como la empresa, la escuela, el hospital (Enriquez, 1992; Powell y DiMaggio, 1999), en cuyo caso se conforman grupos denominados institucionales; cuando ellos no responden específicamente a estas necesidades, son considerados grupos espontáneos. En lo referente a las reglas, se denominan grupos formales cuando estos están directamente regulados, con cierta precisión, por escrito por un conjunto de reglamentos y políticas que establecen recompensas y/o sanciones explícitas; se les asocia directamente con el modelo burocrático (Weber, 1970). Se denominan, por el contrario, grupos informales cuando surgen y se desarrollan, de manera más autónoma, al interior de la organización (Mayo, 1997; Roethlisberger y Dickson, 1967; Homans, 1977). Por otro lado, cuando la razón principal es permanecer juntos, los miembros están centrados más en el grupo y predominan los factores afectivos, a este caso se le conoce como grupo de base; cuando lo esencial es realizar una acción o tomar una decisión, se dice que lo más importante es llevar a cabo una función operativa, y se habla entonces de un grupo centrado en la tarea o de trabajo.

En esta dirección, Anzieu y Martin (20II) proponen cinco categorías fundamentales de grupo: muchedumbre, banda, agrupación, grupo primario y grupo secundario. Debido a su importancia en este trabajo, me referiré solamente a los dos últimos. El grupo primario, pequeño o restringido está constituido, como su nombre lo indica, por un número relativamente reducido de individuos, de tal manera que cada uno puede tener una percepción individual de cada uno de los otros, al mismo tiempo que es percibido por los demás. Se caracteriza por que los fines son comunes y relativamente permanentes; pero lo más relevante es la intensidad con la que se viven las relaciones afectivas, las cuales son cálidas, íntimas, personales, aunque pueden caer fácilmente en el otro extremo y transformarse en relaciones de antipatía, adversidad y enemistad profundas. Se percibe en él un alto grado de solidaridad y de unión moral, aun fuera de sus actividades centrales; sus relaciones suelen ser espontáneas, poco calculadas y frecuentes. Desde un punto de vista psicológico, el 
grupo primario es el resultado de una fusión de individualidades en una sola unidad, de tal manera que la vida común y el fin del grupo pasan a ser el centro y la razón de cada uno de los miembros, y se expresan usualmente de manera plural por medio de un "nosotros". La estructura del grupo primario permite diferenciar sus roles, así como el establecimiento de normas, creencias, lenguaje y códigos particulares. Entre los ejemplos más citados en este tipo de grupo se encuentran la familia y el grupo de amigos, aunque se han venido agregando otros como grupo de vecinos, de paisanos y el compadrazgo en tanto extensión simbólica de la familia. En general, el número de miembros de estos grupos es restringido, aunque existen excepciones como las grandes comunidades religiosas o tribus.

Anzieu y Martin (20II), de acuerdo con Merton (1984), proponen que la organización puede ser considerada como un grupo secundario, constituido por un número mediano o grande de participantes, en el que las relaciones entre ellos son calculadas, impersonales, racionales, formales y contractuales. La organización se forma tomando como referencia las instituciones de la sociedad; así, encontramos por ejemplo las empresas, los hospitales y las universidades, entre otros. Los miembros de la organización se adhieren a los objetivos establecidos por ella, los cuales pueden ser total o parcialmente iguales o diferentes a los de cada integrante. El grado de estructuración que presenta este grupo secundario es el más elevado de las cinco categorías propuestas, estando los roles de los individuos más definidos que en los grupos anteriores. Las actividades se centran más en la realización de la tarea y la dimensión más importante es la funcional; a los grupos operativos que funcionan al interior de la organización se les identifica como equipos de trabajo.

Para efectos de este artículo, entendemos por grupo el conjunto de individuos que se definen como sus integrantes, en donde las relaciones cara a cara son determinantes - perspectiva individual-, es decir, que poseen un fuerte sentimiento de pertenencia y que además son reconocidos y reconocen al resto de los miembros, y generan además un fuerte sentido de unidad - perspectiva grupal-. El grupo debe realizar una tarea central, ligada a un mandato institucional, inducida por un alto nivel de idealidad. En el presente texto, a diferencia de Merton, la organización no es considerada como un grupo sino como una instancia social distinta (Enriquez, 1992). El grupo encuentra en ella un aliado que le facilita su accionar, pero compromete también el logro de sus resultados. Así, el grupo procura y rechaza diversos aspectos del ámbito organizacional.

Del análisis realizado a los grupos queremos destacar los tres factores que consideramos más relevantes en este trabajo; los de orden social, que dotan de sentido a la acción colectiva, los de orden funcional, que proporcionan un halo de racionalidad 
que permite enfrentar tareas de tipo operativo y los de orden afectivo, que generan protección y reconocimiento. El primer orden se inscribe en el ámbito institucional y es trasladado en cierta medida a los otros; el segundo se ubica en el ámbito estructural de la organización, en el que las tareas que se desempeñan implican habilidades técnicas, administrativas y de organización, las cuales se integran a jerarquías formales aparentemente impersonales. El tercer orden se orienta más a las relaciones intersubjetivas intensas que se establecen entre ellos. En este nivel lo más relevante es el grupo en sí mismo. El enfoque psicoanalítico propone no reducir el concepto a aspectos meramente operativos, ya que en una parte importante de los sucesos grupales se encuentran presentes lazos afectivos complejos y profundos de difícil acceso a la esfera de la conciencia de los individuos.

\section{Acerca de la identidad, la pertenencia y la diferenciación}

Consideramos que en el estudio de los grupos resulta esencial abordar el tema de la identidad, ya que el grupo constituye un lugar privilegiado para su construcción. El primer contacto que tiene el individuo al nacer es con un grupo primario, la familia; se trata de su primera experiencia social, en ella empieza a elaborarse su identidad a través de un proceso activo, paulatino, no exento de dificultades y disyuntivas. Posteriormente, en la adolescencia y la adultez, el individuo continuará reelaborando dicha identidad y es precisamente en el seno de los grupos que ese proceso adquirirá los matices propios del ámbito institucional y organizacional al constituir estos espacios sociales y sus representaciones, como la escuela y la empresa, referencias fundamentales de todo individuo, encontrando valores, principios y modelos de comportamiento en los roles sociales. De esta manera, podemos decir que la identidad es un proceso inacabado y dinámico, ya que en diferentes momentos de la edad adulta el individuo seguirá construyéndola en las organizaciones en las que labore así como en diversos grupos de orden político, religioso, deportivo, en los que participe.

Se puede afirmar que una de las principales fuerzas del grupo es la integración, ya que en ella el individuo define su relación también con la organización y la sociedad. El hecho de pertenecer a un grupo proporciona un sentimiento ajeno a la soledad y el caos, que le genera al individuo un sentimiento de fortaleza a la vez que lo sensibiliza a valores que solo tienen sentido en el ámbito de lo colectivo, tal como acontece con la solidaridad, la protección o los fines sociales. Sin embargo, esta manera de integrarse al grupo, de adoptar sus valores, de conformarse y, por decirlo 
de alguna manera, de estandarizarse no significa necesariamente, como ya se mencionó, que la persona desdibuje su personalidad o que pierda lo que lo caracteriza como individuo único. Así como este se sirve del grupo para lograr ciertos fines, o por la imagen grandiosa que le refleja, o por algunos de los otros aspectos antes ya referidos, se sirve de él también para afirmar su propia individualidad.

La integración es un proceso que presenta, grosso modo, dos aristas, la primera es que el hecho de pertenecer a un grupo implica aceptar ciertas limitaciones y normas impuestas por el grupo y, en general, por la sociedad, y una segunda corresponde a las necesidades y aspiraciones que impulsan al individuo a integrarse a un grupo. De ese modo, el grupo impone por un lado restricciones, pero por otro satisface necesidades, lo cual coloca al individuo en una situación de ambigüedad y contradicción, donde en vano trata de articular lógicamente ambas. Si bien, como ya dijimos, el grupo es útil y necesario, dado que el individuo es, en muchas ocasiones, más eficaz y eficiente cuando trabaja en conjunto que cuando lo hace de manera aislada, también es cierto que el grupo representa al mismo tiempo una amenaza para el individuo, ya que puede influir de manera importante en su comportamiento o afectar su individualidad. En una investigación realizada por Anzieu y Martin (20II) a propósito de grupos, los entrevistados respondieron que: "el grupo también presenta ciertos riesgos ya que representa una alienación de la personalidad individual, pues atenta contra la dignidad, la libertad, la autonomía" (Anzieu e Yves Martin, 20II: 20).

La relación del individuo con los otros y con su contexto resulta pues compleja y difícil de entender; su comprensión requiere la realización de esfuerzos importantes para hacer algunos avances. Implica no solo adaptarse al mundo tal como es, sino también tratar de adaptarlo a las propias necesidades, de acuerdo con la forma como se interpreta el mundo. La brecha entre la constatación de la realidad del mundo o, mejor dicho, la percepción que se tenga de dicha realidad, y la apreciación de cómo este debiera ser genera frecuentemente al interior del grupo una serie de malentendidos y conflictos que en ocasiones llevan a la ruptura, dejando a los individuos en una situación de desesperanza, que no siempre puede evitarse.

\section{El caso del equipo universitario de buceo}

\section{Nota metodológica}

Entre las principales herramientas utilizadas en esta investigación quiero destacar el análisis documental, la observación participante y la entrevista semiestructurada. El 
trabajo de campo se realizó por medio de varias visitas exploratorias al grupo en sus instalaciones de trabajo, tanto en oficina como en alberca, en la Universidad Nacional Autónoma de México (UNAM), con el objetivo de introducirnos en la dinámica grupal. ${ }^{3}$ En esta etapa de observación, además, nos dimos a la tarea de conocer a sus dirigentes, así como de identificar a sus principales actores; por ejemplo, a aquellos de mayor jerarquía formal o autoridad moral, o los de mayor antigüedad en el grupo, porque conocen más a fondo su historia y su funcionamiento operativo, así como a aquellos que tienen más influencia en los demás.

La segunda etapa consistió en la elaboración de los guiones para las entrevistas, retomando tanto elementos de la discusión teórica como los resultados más destacados de un breve cuestionario piloto y las características más relevantes observadas en el grupo. En dicho guión se incorporaron aspectos referentes al funcionamiento y los antecedentes del grupo, apoyos académicos, económicos o personales que ha tenido, mecanismos de toma de decisiones, ambiente de trabajo, en particular las relaciones personales, los valores y los conflictos, entre otros; finalmente, inquirimos sobre las fortalezas y debilidades que se perciben en distintos planos de la dinámica grupal -social, organizacional e individual-. Se realizaron 12 entrevistas, entre directivos y miembros del grupo, la mayor parte en el lugar de trabajo, con una duración promedio de una hora cada una, exceptuando la realizada a la coordinadora, de dos horas.

La siguiente etapa consistió en analizar el contenido de las entrevistas, apoyándose también en los documentos de trabajo proporcionados por el grupo, como planes de desarrollo de la Facultad de Ciencias y algunas presentaciones elaboradas por la coordinadora del grupo, en las notas de campo y en conversaciones realizadas en la etapa introductoria. El procesamiento y análisis de la información obtenida a través de las entrevistas se realizó mediante la propuesta del análisis de contenido, entendida en términos generales - de acuerdo con Krippendorff (1990) - como "la técnica destinada a formular, a partir de ciertos datos, inferencias reproducibles y válidas que puedan aplicarse a un contexto". El análisis de contenido utiliza un conjunto diverso de instrumentos que permite realizar interpretaciones en contextos de acción determinados (Creswell: 1998). Se puede decir que, en términos generales, incluye tres grandes etapas (Bardin, 1977): a) el preanálisis, que consiste en un primer

3 Luis Montaño Hirose participó activamente en la concepción y el desarrollo de este estudio. Lucero Jiménez y Ana Lilia Rojas colaboraron en la transcripción de las entrevistas. Agradezco a todos los miembros del ЕвC por su entusiasta participación en este trabajo. Como es habitual, asumo toda la responsabilidad de esta presentación. 
esfuerzo por organizar la información y vislumbrar elementos de sentido otorgado por los sujetos bajo investigación, lo que implica una múltiple lectura atenta de las transcripciones de las entrevistas; $b$ ) el procesamiento y análisis de la información, centrados en la elaboración de las principales categorías; y c) la inferencia y la interpretación, orientadas hacia el esclarecimiento de los sentidos profundos del discurso.

Como último punto se llevó a cabo una reunión de trabajo con la mayor parte de los integrantes del grupo con el objetivo de compartir los análisis realizados a la información recopilada y los resultados obtenidos, lo que en intervención organizacional se denomina la restitución (Herreros, 2002).

\section{El Equipo de Buceo de Ciencias}

\section{Historia y desarrollo del grupo \\ Primera etapa, 1962-1976}

Señalo a continuación las principales etapas por las que ha transitado este grupo desde su inicio hasta la actualidad. El Equipo de Buceo de la Facultad de Ciencias (евС) de la UNAM se formó en 1962 a solicitud de un grupo de profesores con el objetivo de realizar actividades subacuáticas. La propuesta fue dirigida a la Organización de los Estados Americanos (OEA) y la respuesta fue la donación de 20 equipos de buceo para que comenzara sus actividades.

Su principal tarea consistía en organizar cursos de adiestramiento para los estudiantes y proporcionar material para prácticas escolares. Realizó una serie de estudios sobre arrecifes coralinos, principalmente en el sistema arrecifal veracruzano, así como una práctica de campo de biología en Isla Verde, Veracruz, en la que se aplicó el buceo como una herramienta de trabajo. En 1972 el equipo pasa a ser coordinado por un grupo de estudiantes, etapa que desde el punto de vista de algunos entrevistados provocó una disminución notable de la calidad académica.

En 1975 el grupo estaba coordinado por un académico e integrado por I4 estudiantes. En 1976 se asumió como grupo de trabajo académico, coordinado por un biólogo y un asesor académico, con maestría en ciencias. Entre sus objetivos estaba formar personal capacitado en las técnicas de buceo libre y autónomo aplicadas a diferentes áreas científicas, participar en programas de investigación científica y técnica, dentro y fuera de la UNAM, elaborar y desarrollar programas de investigación propios y dar servicios de apoyo a quien lo solicitara. En esta etapa el ввC dependía del Consejo Interdepartamental de la Facultad de Ciencias. 


\section{Segunda etapa, 1976-1996}

Esta etapa se enmarca en la transición al nuevo edificio de la Facultad, abarcando, una primera subetapa hasta 1980. Entonces se formalizan los cursos de buceo al ser reconocidos nacional e internacionalmente por la Federación Mexicana de Actividades Subacuáticas (FMAS) y por la Confederación Mundial de Actividades Subacuáticas (CMAS). De manera simultánea se inicia la capacitación de sus integrantes como instructores de buceo. Esto repercutió en el desarrollo de una escuela de buceo con orientación académica. Desde entonces han continuado impartiéndose cursos en diferentes niveles.

En este periodo el grupo estuvo constituido por estudiantes organizados en tres coordinaciones -académica, técnica y deportiva-, aún bajo la asesoría del maestro en ciencias. La labor académica del grupo se promovió mediante ciclos de conferencias y con la organización de prácticas de campo, prosiguiendo con los estudios sobre arrecifes coralinos. Al finalizar esta subetapa, en I98I, el ЕBC pasa a formar parte del Departamento de Biología.

En 1984, tras un breve periodo en el que se puso énfasis en la actividad deportiva, se retomó nuevamente la orientación académica al reanudarse el proyecto sobre las comunidades arrecifales. En esta nueva etapa, que abarca hasta 1995, se continuó con las prácticas de campo y con la organización de ciclos de conferencias. El grupo se encontraba integrado por académicos y alumnos. Se inició un programa de enseñanza de buceo científico que incluía cursos intermedios y avanzados, promoviendo la capacitación de los alumnos en técnicas de investigación subacuática. Estos se encontraban vinculados con las prácticas de campo, apoyando en labores de muestreo, colecta y toma de datos durante sus salidas de trabajo.

\section{Tercera etapa, I996 a la fecha}

La etapa más reciente, desde 1996 a la fecha, se caracteriza por un proceso de consolidación del proyecto de desarrollo del EBC, impulsando la implementación de un programa académico de buceo científico en la Facultad de Ciencias. En la actualidad el grupo está integrado por 14 miembros, entre ellos se encuentran la coordinadora general, un asesor académico, un asesor médico, un técnico en urgencias médicas y un conjunto de instructores llamados "monitores". 


\section{Principales funciones del EBC}

Entre las principales tareas asignadas al евс están las de apoyar a la comunidad académica del Departamento de Biología de la Facultad de Ciencias para el desarrollo de proyectos de investigación, actividades docentes y de divulgación, que requieran el buceo como una herramienta de trabajo. Este respaldo consiste principalmente en la colecta de organismos, asistencia logística durante salidas de campo, asesorías técnicas, apoyo bibliográfico y préstamo de equipo de buceo y material de colecta para la realización de prácticas. Los integrantes del grupo están conscientes de que sus objetivos deben de apegarse a los de la universidad en tanto institución social: "Los objetivos que debe tener el евC deben ser los mismos que tiene la universidad, divulgación de la ciencia, investigación y docencia, no puedes salirte de estos tres":

Otra de sus actividades consiste en impartir cursos de capacitación en diferentes niveles y orientación, dirigidos a estudiantes y profesores enfocados principalmente a la utilización del buceo como una metodología de trabajo de campo. En el caso de los estudiantes se pretende fomentar este aprendizaje como un complemento a su formación académica, como biólogos u otras carreras afines, y en general a la comunidad universitaria cuyo interés se enfoque hacia las ciencias acuáticas y/o a la preservación de los recursos marinos. El préstamo de material y equipo de buceo es otro apoyo que ofrece el вBC, dependiendo de su disponibilidad.

\section{Análisis de la información}

Actualmente, el ЕвC se caracteriza por ser un grupo formado en general por jóvenes cuyas edades oscilan entre 25 y 35 años, la mayoría son universitarios que se encuentran estudiando o elaborando la tesis para obtener el grado académico, solo dos de ellos mantienen una relación de trabajo formal con la UnAm como académicos. El евс se define a sí mismo como un grupo autosustentable que genera además recursos económicos por medio de los cursos que imparte a través de la Secretaría de Educación Abierta y Continua de la Facultad de Ciencias. El equipo de buceo con el que cuenta actualmente ha sido obtenido en gran medida por vía de los ingresos provenientes de los cursos que ofrece. Cabe mencionar que los integrantes del grupo realizan el trabajo de forma totalmente voluntaria, sin percibir compensación económica alguna.

El евс es un grupo altamente demandante; la naturaleza misma de la tarea -con un alto nivel de riesgo-, la implicación personal en términos institucionales 
y el compromiso afectivo representan un arduo trabajo que requiere reuniones frecuentes, de dos a cuatro veces por semana, y la consecuente inversión de una parte importante de tiempo; así comenta uno de los miembros: "Se vuelve pesado estar viniendo viernes, sábado, domingo y lunes, los cuatro días, porque a veces sí te cansas físicamente, pero cuando estoy dando mi clase digo: valió la pena todo ese esfuerzo..., la satisfacción es más que el cansancio."

Para analizar la información obtenida propongo tres ejes principales de reflexión: ${ }^{4}$

a. Institución/organización y grupo. En este eje se considera a la universidad como institución, a la UNAM/Facultad de Ciencias como la organización y al EBC como el grupo. Se pretende estudiar las particularidades de dicha relación.

b. Grupo e individuo. En esta sección se aborda la relación del евC con sus integrantes, considerados a nivel individual, tomando en consideración sus diversas trayectorias y particularidades.

c. Grupo y equipo. El primero es entendido como el conjunto de individuos que se reúnen por aspectos afectivos, y el segundo, como la reunión de compañeros que se encuentran de manera formal para desempeñar tareas o actividades específicas. Esta categoría pretende analizar los diferentes roles que juega cada uno, destacando la dificultad que existe en ocasiones para diferenciarlos, lo que crea una situación de confusión acerca del sentido del grupo e interfiere en la toma de decisiones (Bion, 196I).

De esta manera, si bien al interior de cada eje analítico se pueden observar aspectos que se complementan entre sí - convergencias-, también se detectan otros que en ocasiones coinciden menos o bien se contradicen abiertamente, dando lugar al surgimiento de divergencias.

\section{Institución/organización y grupo}

Una de las principales preocupaciones que tiene actualmente el ввC es la falta de reconocimiento formal como grupo de trabajo ante la UNAM, y de manera más

4 La conformación de estos ejes analíticos la realicé en función de la información obtenida y de su relevancia. Así, decidí incluir en un mismo apartado la relación del grupo con la institución y la organización, en los términos teóricos tratados en el primer apartado. Por otro lado, consideré importante incorporar una breve reflexión del vínculo individuo/grupo por la diversidad y relevancia de situaciones particulares. 
específica, ante la Facultad de Ciencias. Como se menciona en la historia del eBC, este ha tenido diversos estatus como servicio que otorga dicha Facultad. Así lo expresa un integrante: "Tiene reconocimiento oficial; sin embargo, no está reconocido como un laboratorio, digamos, lo tienen al mismo nivel que un grupo estudiantil."

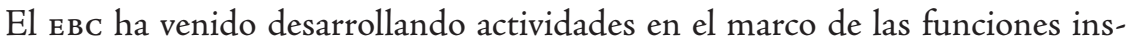
titucionales de la UNAM, en este caso principalmente la docencia; en cuanto a la investigación, ha participado en algunos proyectos apoyando prácticas de campo y colecta de especímenes. Un miembro expresa el objetivo del евс: "lo que nos interesa es que los alumnos aprendan las técnicas básicas para llevar a cabo su propia investigación, nosotros en realidad no somos generadores de conocimiento".

Sin embargo, existen actividades en las cuales no pueden participar plenamente; al no tener el reconocimiento formal, no son sujetos, por ejemplo, de recibir cierto tipo de apoyos, entre otros los económicos, por parte de la UNAM y de otras organizaciones: "podría generar proyectos de investigación, cosa que ahorita no se puede porque como grupo estudiantil no puede atraer un proyecto como tal".

Sin embargo, sin negar el ámbito emocional afectivo, el grupo está consciente de que es parte de la universidad. De hecho, podría considerarse que dicha alusión es tanto institucional como organizacional; se refieren a ella tanto como componente idealizado e imaginario del orden social a la vez que como experiencia próxima y concreta, la UNAM: "Nos involucramos no solo por nosotros, no solo por nuestra amistad, sino por la universidad, porque sabemos que lo estamos haciendo por una institución."

El EBC mantiene relaciones internas en la UNAM a través de tres instancias que son la Facultad de Ciencias, que le tiene asignado un espacio físico en donde se realizan las reuniones de trabajo y guarda del equipo de buceo; en este mismo rubro, al EBC le han sido aprobados diplomados de Buceo Científico y de Liderazgo en concordancia con otras instancias universitarias. Una segunda corresponde a la Asociación de Actividades Subacuáticas de la UnAm (AASUnAm), la cual se encarga de regular el acceso a la alberca para la realización de las clases prácticas; la tercera corresponde a la Dirección General de Actividades Deportivas y Recreativas (DGAdyr) que se encarga de regular y promover las actividades deportivas al interior de la UNAM.

En este apartado consideramos importante mencionar, además de las relaciones con la UNAM, otras que mantiene el вBC con el exterior, como el vínculo con la Federación Mexicana de Actividades Subacuáticas (fMAs) que es, como ya se mencionó, el organismo que certifica a los buceadores de manera formal en México y que funciona en concordancia con la Confederación Mundial de Actividades Subacuáticas (cmas). 
La relación entre el eBC y la Facultad de Ciencias presenta un doble registro, uno formal y otro informal. El primero, por su propio origen, es en ocasiones poco claro, posee un mínimo de sustento documental, y a pesar de ello el grupo se apega estrechamente a su referencia institucional universitaria. El segundo, el informal, implica la instauración de formas de organización en las que la afectividad juega un papel central.

\section{Grupo e individuo}

En este punto nos centramos básicamente en el interés de los individuos por pertenecer al grupo. La participación de la mayoría de ellos responde principalmente a dos tipos de intereses. Los primeros, de orden institucional o social, se inclinan a aspectos centrales de las funciones esenciales de universidad, en especial la impartición de cursos y el desarrollo de actividades relacionadas con la investigación. Varios de los miembros se expresan en este sentido:"yo disfruto más enseñando y dando clases y al ver que el alumno realmente logra sus objetivos", o bien, "me gusta la academia, me gusta leer, aprender, enseñar; entonces, esas cosas me gustan mucho, como dar clase."

Algunos otros esgrimen razones que colindan con el esparcimiento o el deporte mientras que otros más lo hacen por el interés de proteger los ambientes naturales: "mi principal objetivo de estar aquí y de bucear es precisamente conocer estos ambientes (subacuáticos) para poder conservarlos, pues muchos de ellos nos brindan servicios al hombre o recursos", o bien,"Desde la facultad buscaba ser bióloga marina porque me encantaba todo lo que tenía que ver con el mar; entonces, siempre he ido en esa dirección."

Un tercer tipo de interés responde a motivos afectivos, de orden simbólico, frecuentemente inconscientes; algunos miembros buscan un reconocimiento de los compañeros del grupo o de los alumnos, posiblemente, en ocasiones, para compensar lo que no reciben en otros ambientes -laboral, familiar, etc.-. Ejemplo de motivos afectivos es la siguiente expresión: "Lo más importante es la actitud, porque las habilidades, como apoyar en cuestiones teóricas o prácticas, así como que hacer más ejercicio, es más fácil, pero la parte emocional y la parte de madurez es como la más importante."

En lo que se refiere a motivos simbólicos se puede destacar el ascenso en la jerarquía social:"pertenecer al equipo es muy importante, pero hay mucha gente que parece que ser parte de él es ultra plus, así como el sueño de alguna persona. Y ya que estás dentro parece que es una forma de elevarse hasta una punta social importante." 
Para otros miembros, el grupo representa un refugio, esto probablemente debido a que en otros ambientes no han obtenido los logros esperados; en este caso, en el grupo encuentran apoyo y seguridad. Recordemos que una parte importante de los miembros del grupo son jóvenes que se encuentran en una etapa de búsqueda de referencias sociales, por ello se procura resguardar esta identidad colectiva, aun a costa de la individualidad: "aquí no trabajamos de manera individual, trabajamos todos juntos; entonces, es importante que haya una buena relación con todos".

Las diferentes personalidades de los miembros son motivo de conflictos, en ocasiones agudos, en el interior del grupo; ello mismo, por otro lado, posibilita una mejor vinculación entre ellos para desempeñar sus actividades: "he aprendido muchísimo dentro del equipo, la parte del liderazgo de cómo entender a las gentes y cómo tratar a las personas para que podamos cumplir".

Como se ha mencionado, el individuo tiene interés en pertenecer a un grupo por todos los beneficios que recibe, pero también por otro lado observa algunos inconvenientes al vincularse a él de manera tan cercana: "creemos que la estancia dentro del equipo sí necesita como una fortaleza física y mental para poder aguantar estar cada ocho días, estar conviviendo entre nosotros".5

El hecho de enfrentar cambios ocasiona temores, como es el caso que se presenta con la permanencia de los miembros o la entrada de nuevos. En los grupos pequeños la relación entre los individuos resulta cercana y cotidiana, y cuando se percibe algún cambio, los individuos manifiestan preocupación, e incluso angustia, y así lo expresan: "quiero que los que estén ahora, se queden aquí para siempre y no cambien nunca”.

El ingreso de nuevos miembros es, por lo tanto, motivo de grandes temores: "hablando de integrar a más miembros, creo que es por miedo también, es un grupo cerrado".

\section{Grupo/equipo de trabajo}

Ambas figuras están intrínsecamente imbricadas (Montaño, 2014). Por grupo me refiero al conjunto de individuos cuya relación principal es de orden intersubjetivo, es decir, expresan vínculos afectivos profundos. Uno de los integrantes lo manifiesta así: "el apoyo más importante creo que es el afectivo, pues saber que tienes a

5 Esta expresión me recuerda la sentencia de Sartre: "El infierno son los otros", en A puerta cerrada. 
tu lado no solo a tus compañeros de buceo, sino a amigos, como a toda tu familia, y toda esa parte emocional de tener muchos amigos y saber que puedes confiar en cualquiera de ellos".

Por otro lado, el grupo, entendido como se acaba de mencionar, también muestra algunas limitaciones cuando el hecho de ser amigos impide hacer reconocer los errores a los demás: "Se cometió una falta... son tus compañeros, son tus amigos, pero aquí estás por un grupo, no estás por ser amigos".

Con frecuencia se confunde el ser un grupo y el ser un equipo; el equipo está enfocado a la tarea, los miembros son compañeros y no debería ser tan importante la amistad o la diversidad de personalidades. Un entrevistado se expresa al respecto: "hablando de fraternidad, que no se confunda con amistad, porque una cosa es que seamos amigos y otra cosa es de que yo te apoye porque somos parte del mismo grupo; entonces podemos ser muy amigos o muy enemigos pero cuando tenemos que hacer cosas somos parte de lo mismo".

El trabajo en equipo es diferente al de un grupo de amigos. Así lo manifiesta un miembro: "durante el trabajo yo creo que el trato sí debe ser más profesional, más organizado, más estructurado y dejar a un lado la amistad".

En esta categoría se presentan conflictos relacionados entre la jerarquía formal de puestos y la dinámica afectiva del grupo: "sí, soy tu jefe, pero también soy tu amigo, entonces hay veces que te trato como jefe, hay veces que te trato como amigo y entonces de repente tú ya no sabes si eres mi subordinado, mi amigo, o mi qué".

Así como encontramos divergencias entre grupo y equipo, también las encontramos dentro del mismo equipo. Hacia fuera del ввс, todos los miembros se ven como una unidad sólida, pero al interior se constatan diferencias; lo comenta un miembro: "el discurso iba como hacia 'todos somos parte del equipo'; enfrente de los alumnos todos somos instructores y somos un bloque, y así nos vamos a comportar, pero aquí, dentro del equipo, sí hay jerarquías,"

Finalmente, como ya se mencionó, el grupo tiene un gran interés en ser reconocido formalmente por la UNAM, pero también señala su abierto temor a las limitaciones que podría enfrentar el desarrollo de su rol institucional, al tener que apegarse a las reglas que la universidad le impone. Si bien el deseo de pertenencia es fuerte, también lo es el temor a la pérdida de autonomía: ser más equipo podría significar ser menos grupo. 


\section{Conclusiones}

El estudio del grupo constituye en la actualidad un gran reto; relegado en ocasiones a una instancia de segundo orden, es considerado como un ámbito social menor, cada vez menos importante ante la creciente individualidad y racionalidad inducidas por la modernidad avanzada. La vida afectiva y simbólica constituye en esta visión un obstáculo al desarrollo armónico al involucrar aspectos de tipo "irracional” en el comportamiento de los actores. En todo caso, se asume generalmente que el grupo se presenta en casos extremos, alejados del mundo de la racionalidad operativa, como en el caso de grupos de estudiantes o bien los grupos informales al interior de espacios formalizados; suele referirse a ellos cuando son aceptados, en tanto equipos de trabajo con capacidad para explotar habilidades individuales distintas, necesarias para la realización efectiva de tareas específicas.

A la crítica anterior es preciso agregar que la mayoría de los estudios de grupo corresponde a la tradición de la metodología cualitativa, la cual no ha sido plenamente aceptada, sobre todo por aquellos que la consideran una modalidad de estudio de realidades totalmente circunscritas cuyos resultados no son en absoluto generalizables. En efecto, la metodología cualitativa ha sido cuestionada en amplias esferas académicas por una supuesta falta de rigor científico, destacándose de manera central su indisposición hacia la generalización. Los estudios de caso, propios de dicha aproximación metodológica, son condenados a permanecer en el espacio social acotado en el que se desarrollaron, o bien a la ardua tarea, casi interminable, de acumular una cantidad enorme de casos a los que además hay que vincular de tal manera que proporcionen un panorama coherente que permita visiones de tipo genérico. De esta manera, se pierde de vista la posibilidad de aprovechar las ventajas del estudio de caso, y principalmente su nivel de profundidad, ya que la capacidad de los estudios cuantitativos resulta muy limitada al explorar el sentido otorgado por los actores a sus propias acciones. A ello resulta importante además añadir la capacidad que tiene la perspectiva cualitativa de realizar generalizaciones de tipo analítico (Yin, 2009; Giménez, 2012; Rendón, 2014).

Con respecto a estas dos objeciones, en este trabajo se asume una perspectiva distinta. Se postula, primero, que el grupo es un espacio social preponderante y no secundario, tanto para la acción individual como para la organizacional y social; segundo, que el grupo se entreteje siempre, en mayor o menor medida, con el equipo, generando convergencias y divergencias que son intensamente vividas por el individuo de manera contradictoria, así como amplios y potentes consensos pero también graves y destructivos conflictos; tercero, que el grupo constituye un 
dispositivo privilegiado para la reelaboración constante de la identidad del actor; y cuarto, que el estudio de caso, inscrito en la metodología cualitativa, proporciona elementos de referencia fundamentales para la comprensión de otros casos que se encuentran aparentemente alejados.

De esta manera, el estudio de un grupo como el que hemos emprendido no debe limitarse, por dar un ejemplo, al caso de los grupos universitarios de buceo en países latinoamericanos, sino que debe pensarse que algunas de sus aportaciones permiten realizar, así sea por simple contraste, reflexiones importantes acerca de la importancia de los grupos en otros ámbitos, por ejemplo, en las universidades públicas mexicanas, con la incorporación de los cuerpos académicos. La capacidad de los grupos de potenciar y obstaculizar, mediante la vida afectiva y sus múltiples referencias institucionales, las funciones principales de cualquier organización constituye ya una poderosa hipótesis de trabajo que nos permite generar una reflexión en ámbitos institucionales diversos. Podemos decir que los grupos en las organizaciones desarrollan de manera intensa su razón de ser cuando se intersecan con las actividades sustantivas que legitiman de manera institucional sus funciones sociales primordiales.

\section{Bibliografía}

Aebischer, Verena y Dominique Oberlé (1998), Le groupe en psychologie social, París, Dunod.

Amado, Giles y Andé Guittet (1975), Dynamique des communications dans le groupe, París, Armand Colin.

Anzieu, Didier y Jacques Yves Martin (20II), La dynamique des groupes restreints, París, Quadrige, Presses Universitaires de France.

Bardin, Laurence (1977), L’analyse de contenu, París, Quadrige, Presses Universitaires de France.

Bateson Gregory, Don D. Jackson, Jay Haley y John Weakland (1956),"Toward a Theory of Schizofrenia," Behavioral Science, I(4), pp. $251-254$.

Berger, Peter L.y Thomas Luckmann (1968), La construcción social de la realidad, Buenos Aires, Amorrortu.

Bion, Wilfred (196I), Experiences in Groups, Londres, Routdlege.

Bleger, José (1993), "El grupo como institución y el grupo en las instituciones", en René Kaës (coord.), La institución y las instituciones. Estudios psicoanalíticos, Buenos Aires, Paidós, pp. 68-83. 
Creswell, John W. (1998), Qualitative inquiry and research design: Choosing among five traditions, Thousand Oaks, Sage.

Cuevas Burgos, Manuel y Rodrigo Guerra Arrau (2010), "Vínculo y sufrimiento institucionales. Discusión de conceptos a partir de un estudio de caso", Praxis. Revista de Psicología, I7, pp. 23-50.

DiMaggio, Paul J. y Walter W. Powell (1999), "Retorno a la jaula de hierro: el isomorfismo institucional y la racionalidad colectiva en los campos organizacionales", en Walter W. Powell y Paul DiMaggio (comps.), El nuevo institucionalismo en el análisis organizacional, México, Colegio Nacional de Ciencias Políticas y Administración Pública/Universidad Autónoma del Estado de México/Fondo de Cultura Económica, pp. I04-I25.

Enriquez, Eugène (1992), Lorganisation en analyse, París, Presses Universitaires de France.

Enriquez, Eugène (1997), Les jeux du pouvoir et du désir dans l'entreprise, París, Desclée de Brouwer.

Freudenberger, Herbert J. (1974), "Staff Burn-Out", Journal of Social Issues, 30(I), pp. 159-165.

Freudenberger, Herbert J. (1980), Burnout: The high cost of high achievement, Nueva York, Doubleday.

Friedland, Roger y Robert R. Alfort (200I), "Introduciendo de nuevo a la sociedad: símbolos, prácticas y contradicciones institucionales", en Walter W. Powell y Paul DiMaggio (comps.), El nuevo institucionalismo en el análisis organizacional, México, CNCPyA /UAEMex/FCE, pp. 294-329.

Giménez, Gilberto (2012),"El problema de la generalización en los estudios de caso", Cultura y representaciones sociales, 7(12), pp. 40-62.

Herreros, Gilles (2002), Pour une sociologie d'intervention, París, Éditions érès. Homans, Georges (1977), El grupo bumano, Buenos Aires, Eudeba.

Kaës, René (2004), “Complejidad de los espacios institucionales y trayectos de los objetos psíquicos", Psicoanálisis Asociación Psicoanalítica de Buenos Aires, 26(3), pp. 655-670.

Kaës, René (2005), "Souffrance et psychopathologie des liens institués. Une introduction”, en René Kaës (coord.) Souffrance et psychopathologie des liens institués, París, Dunod, pp. I-47.

Krippendorff, Klauss (1990), Metodología del análisis de contenido: Teoría y práctica, Barcelona, Paidós. 
Lapassade, Georges (2006), Groupes Organisations Institutions, París, Editorial Economica, Anthropos.

Maisonneuve, Jean (1980), La dinámica de los grupos, Buenos Aires, Nueva Visión. Mayo, Elton (1997), Problemas sociales de una civilización industrial, Buenos Aires, Nueva Visión.

Merton, Robert K. (1984), Teoría y estructuras sociales, México, FCE.

Michels, Robert (1976), Los partidos políticos. Un estudio sociológico de las tendencias oligárquicas de la democracia moderna, 2 tomos, Buenos Aires, Amorrortu.

Montaño Hirose, Luis (2014), "Léquipe-groupe: une unité complexe. L'expérience d'une équipe de plongée scientifique au Mexique", Nouvelle Revue de Psychosociologie, 18, pp. 223-240.

Montaño Hirose, Luis (2015), Administración y estudios organizacionales. Trayectorias, entrecruces y desafios, 40 aportaciones de la UAM-Iztapalapa, México, Universidad Autónoma Metropolitana.

Rendón Cobián, Marcela (2007), Dinámica organizacional y referentes institucionales. El caso de un pequeño hospital de propiedad familiar, México, Universidad Autónoma Metropolitana-Iztapalapa.

Rendón Cobián, Marcela (2014),"La relevancia del contexto en el análisis organizacional. Tradición y modernidad en una empresa familiar en Chiapas", Nova Scientia, 6(12), pp. 321-355.

Olmsted, Michael S. (1979), El pequeño grupo, Buenos Aires, Paidós.

Pagès, Max (1968), La vie affective des groupes. Esquisse d'une théorie de la relation bumaine, París, Dunod.

Pichon-Rivière, Enrique (1983), El proceso grupal. Del psicoanálisis a la psicología social (I), México, Nueva Visión.

Rohetlisberger, Fritz J.y William J. Dickson (1967), Management and the Worker, Massachusetts, Harvard University Press.

Universidad Nacional Autónoma de México, Facultad de Ciencias Plan de desarrollo 2006-2010, México.

Universidad Nacional Autónoma de México, Facultad de Ciencias, Plan de desarrollo 2010-2014, México.

Weber, Max (1970), Economía y sociedad, México, FCE.

Yin, Robert K. (2009), Case Study Research. Design and Methods, $4^{\mathrm{a}}$ ed., Londres, Sage. 
Marcela Victoria Rendón Cobián realizó la licenciatura en Contaduría Pública en la Universidad Nacional Autónoma de México, obtuvo el diploma en Estudios Avanzados por la Universidad de París Ix-Dauphine, Francia, con la especialidad en Estructuras y Cambios Humanos en las Organizaciones, y cursó el doctorado en Estudios Organizacionales en la Universidad Autónoma Metropolitan-Iztapalapa, donde obtuvo la medalla al mérito académico. Es autora de los libros Organización y cultura. Tradición, poder y modernidad en México, así como Dinámica organizacional $y$ referentes institucionales. El caso de un pequeño hospital de propiedad familiar. Ha publicado diversos artículos referentes al análisis organizacional. Se desempeña como profesora titular de tiempo completo en la Universidad Autónoma Metropolitana, Unidad Iztapalapa, desde 1981. Forma parte del Área de Investigación Organización y Sociedad y del Cuerpo Académico del mismo nombre, así como del Núcleo Básico del Programa de Posgrado en Estudios Organizacionales y del Posgrado en Estudios Sociales. Actualmente es miembro del Sistema Nacional de Investigadores, del Consejo Nacional de Ciencia y Tecnología (Conacyt).

Citar como: Rendón Cobián, Marcela Victoria (2016), "La relevancia del grupo en la dinámica institucional: un equipo de buceo universitario," Iztapalapa. Revista de Ciencias Sociales y Humanidades, núm. 81, año 37, julio-diciembre de 2016, ISSN: 2007-9176; pp. 4I-65. Disponible en <http://revistaiztapalapa.izt.uam.mx/index.php/izt/issue/archive >. 